\title{
REFERENCES
}

1. K. D. Arrow, T. E. Harris, and J. Marschak, Optimal inventory policy, Econometrica 19, 250-272 (1951)

2. R. Bellman, Dynamic programming, Princeton University Press, Princeton, New Jersey, 1957

3. R. Bellman, Dynamic programming and the computational solution of feedback design control processes, Conference on Computers in Control, AIEE, October 16-18, 1957, Atlantic City, New Jersey

4. R. Bellman, Some new techniques in the dynamic programming solution of variational problems, Quart. Appl. Math., 16 295-305 (1958)

5. R. Bellman, On a dynamic programming approach to the caterer problem-I, Management Science 3, 270-278 (1957)

6. R. Bellman, I. Glicksberg and O. Gross, On the optimal inventory equation, Management Science 2, 83-104 (1955)

7. A. Dvoretzky, J. Kiefer and J. Wolfowitz, The inventory problem, I, II, Econometrica 20, 187-222 (1952)

8. T. M. Whitin, The theory of inventory management, Princeton University Press, Princeton, New Jersey, 1953

9. H. Scarf, S. Karlin and K. J. Arrow, Studies in the mathematical theory of inventory and production, Stanford Univ. Press, 1958

\section{AN ELEMENTARY DISCUSSION OF DEFINITIONS OF STRESS RATE*}

BY WILLIAM PRAGER (Brown University)

1. Introduction. The simplest constitutive equation considered in the theory of plasticity only involves the tensors of stress and rate of deformation and described rigid, perfectly plastic behavior. When elastic effects are to be included in the analysis, this equation is assumed to apply to the plastic part of the rate of deformation, to which an elastic part must be added before the total rate of deformation is obtained. In a similar manner, a simple constitutive equation for a viscoelastic material can be established by adding an elastic rate of deformation to the rate of deformation of a viscous fluid. In both cases the elastic rate of deformation is usually written as a function of an appropriately defined rate of stress.

This stress rate must obviously satisfy the following condition: if a stressed continuum performs a rigid body motion and the stress field is independent of time when referred to a coordinate system that participates in this motion, the stress rate vanishes identically. As is readily seen, this restriction is not severe enough to lead to a unique definition of stress rate. Indeed, from one definition that satisfies this condition another one may be obtained by adding terms that contain the rate of deformation. In a rigid body motion, these terms vanish, and the second definition reduces to the first. Since the condition imposed on the definition of stress rate only concerns rigid body motions, it will be satisfied by the second definition if it is satisfied by the first. As a consequence of this freedom of choice, many definitions of stress rate are found in the literature.

A similarly embarrassing choice offers itself when one attempts to define finite strain. Potentially, any tensor formed from the displacement gradients qualifies as strain tensor if it vanishes identically for all rigid body motions. Depending on the field of

*Received Feb. 6, 1960. This paper is based on a report distributed under Contract Nonr 562(10) between the Office of Naval Research and Brown University. 
application, one or the other definition of strain may be more convenient to use. Whereas the relative merits of the various definitions of strain are well understood, those of the various definitions of stress rate have not been discussed in similar detail. Moreover, some of these definitions are usually presented in a manner that presupposes familiarity with general tensor calculus. In this paper, only Cartesian tensors are used; the principal definitions of stress rate found in the literature are derived in an intuitive manner, and their suitability for the mathematical description of elastic, plastic behavior is discussed.

2. Notations and basic relations. Let $t$ denote the time and $x_{i}(i=1,2,3)$ a fixed system of rectangular Cartesian coordinates, and denote partial differentiation with respect to these independent variables by the operators $\partial_{0}$ and $\partial_{i}$, respectively.

A tensor of the second order, $T_{i i}$, can be written as the sum of its symmetric and antisymmetric parts, which will be denoted as follows:

$$
\begin{aligned}
& T_{(i i)}=\frac{1}{2}\left(T_{i i}+T_{i i}\right), \\
& T_{(i j]}=\frac{1}{2}\left(T_{i i}-T_{i i}\right) .
\end{aligned}
$$

If $v_{i}\left(x_{1}, x_{2}, x_{3}, t\right)$ is the time-dependent velocity field of a continuum, the rate of deformation is defined as the symmetric part $\partial_{(i} v_{i}$ of the tensor $\partial_{i} v_{i}$, and the rate of rotation as its antisymmetric part $\partial_{[i} v_{i 1}$.

The acceleration of a typical particle is the material derivative $v_{i}^{\cdot}$ of its velocity, which is defined by

$$
v_{i}^{\bullet}=\partial_{0} v_{i}+v_{i} \partial_{i} v_{i},
$$

where the repeated subscript indicates summation in the usual manner. The material derivative of the infinitesimal vector $d s_{i}$ joining adjacent particles is

$$
\left(d s_{i}\right)^{\bullet}=\partial_{j} v_{i} d s_{i} .
$$

Because it will suggest a possible definition of stress rate by analogy, we consider the second rate of deformation. If $d s_{i}$ and $\delta s_{i}$ are the infinitesimal vectors joining the typical particle to two neighboring particles, it follows readily from (3) that the material derivative of their scalar product is

$$
\left.\left(d s_{i} \delta s_{i}\right)^{\bullet}=2 \partial_{(i} v_{i}\right) d s_{i} \delta s_{i} .
$$

Forming the material derivative of (4), we obtain, after some simplifications,

$$
\left.\left(\begin{array}{ll}
\left(s_{1} \delta s_{i}\right.
\end{array}\right)^{\bullet}=2\left[\left(\partial_{(i} v_{i)}\right)^{\bullet}+\partial_{i} v_{k} \partial_{(k} v_{i)}+\partial_{j} v_{k} \partial_{(k} v_{i}\right)\right] d s_{i} \delta s_{i} .
$$

Since the coefficient of $2 d s_{i} \delta s_{i}$ in (4) is called the rate of deformation, the corresponding coefficient in (5), i.e. the contents of the bracket, may well be called the second rate of deformation. Higher rates of deformation have been discussed by Rivlin and Ericksen $[1]^{*}$.

The oriented surface element formed by the infinitesimal vectors $d s_{i}$ and $\delta s_{i}$ is defined as

$$
d A_{i}=\epsilon_{i j k} d s_{i} \delta s_{k},
$$

where $\epsilon_{i j k}$ is the alternating tensor. It is readily verified by means of (3), that the material

*Numbers in brackets refer to the Bibliography at the end of the paper. 
derivative of this surface element can be written as

$$
\left(d A_{i}\right)^{\bullet}=\partial_{i} v_{i} d A_{i}-\partial_{i} v_{i} d A_{i}
$$

(see, for instance, [2, p. 598]).

3. Jaumann's definition. If $\sigma_{i j}\left(x_{1}, x_{2}, x_{3}, t\right)$ denotes the time-dependent stress field in the continuum, the material derivative

$$
\sigma_{i j}^{\cdot}=\partial_{0} \sigma_{i j}+v_{k} \partial_{k} \sigma_{i j}
$$

indicates the time rate of change of a typical stress component at a particle of the continuum, the stress tensor being referred to the fixed coordinate system.

When the continuum and its stress field perform a rigid body motion other than a mere translation, $\sigma_{i j}^{\cdot}$ does not vanish identically. Accordingly, (8) is not acceptable as a definition of the stress rate for use in constitutive equations. A suitable definition is obtained as follows.

Let $P, Q_{1}, Q_{2}, Q_{3}$ be neighboring particles such that the lines $P Q_{\alpha}(\alpha=1,2,3)$ indicate a system of principal axes of the rate of deformation at the particle $P$ and the time $t$, and denote the positions of these particles at the time $t+d t$ by $P^{\prime}, Q_{1}^{\prime}, Q_{2}^{\prime}, Q_{3}^{\prime}$. As the shear rates for the principal axes of the rate of deformation vanish, the lines $P^{\prime} Q_{\alpha}^{\prime}$ are orthogonal. The stress rate could be so defined that it vanishes if the stress tensor at $P$ has the same components with respect to the axes $P Q_{\alpha}$ at the time $t$ as the stress tensor at $P^{\prime}$ has with respect to the axes $P^{\prime} Q_{\alpha}^{\prime}$ at the time $t+d t$.

Let $\lambda_{i}^{\alpha}$ be the unit vector of the direction $P Q_{\alpha}$ and $\lambda_{i}^{\alpha}+\left(\lambda_{i}^{\alpha}\right)^{\circ} d t$ that of the direction $P^{\prime} Q_{\alpha}^{\prime}$. Since the axes $P Q_{\alpha}$ have the rate of rotation $\partial_{1 i} v_{i 1}$, we have

$$
\left(\lambda_{i}^{\alpha}\right)^{\bullet}=\partial_{\{i} v_{i 1} \lambda_{i}^{\alpha} .
$$

Now, a typical component of the stress tensor $\sigma_{i}$ with respect to the axes $P Q_{\alpha}$ is given by the expression $\sigma_{i j} \lambda_{i}^{\alpha} \lambda_{i}^{\beta}$. According to (9), the material derivative of this expression is

$$
\left(\sigma_{i,} \lambda_{i}^{\alpha} \lambda_{i}^{\beta}\right)^{\cdot}=\left(\sigma_{i i}^{\cdot}+\sigma_{k i} \partial_{{ }_{i}} v_{k 1}+\sigma_{i k} \partial_{{ }_{i j}} v_{k 1}\right) \lambda_{i}^{\alpha} \lambda_{i}^{\beta} .
$$

If the material derivatives of all these stress components are to vanish, the parenthesis in (10) must vanish. The expression in the parenthesis thus constitutes a suitable definition of stress rate. On account of the symmetry of the stress tensor and the antisymmetry of the rate of rotation tensor, this definition, which is due to Jaumann [3], may be written as

$$
\sigma_{i j}^{\prime}=\sigma_{i j}^{\cdot}-\sigma_{i k} \partial_{\lfloor k} v_{i\rfloor}-\sigma_{i k} \partial_{\lfloor k} v_{i !} .
$$

Jaumann's work does not seem to be well known: the definition (11) is frequently used in the recent literature [4] without reference to Jaumann.

Since Jaumann's stress rate $\sigma_{i j}^{\prime}$ measures the rate of change of the stress components with respect to a rectangular Cartesian system that participates in the rotation of the material, $\sigma_{i j}^{\prime}=0$ implies that the invariants of the stress tensor are stationary.

4. The definition of Cotter and Rivlin. Let $l_{i}^{\alpha}(\alpha=1,2,3)$ be three linearly independent vectors emanating from the typical particle $P$ at the time $t$. During the time interval $(t, t+d t)$, the neighborhood of $P$ undergoes the infinitesimal affine transformation specified by tensor $\partial_{i} v_{i}$. If the vectors $l_{i}^{\alpha}$ are subjected to the same transformation, we have, in analogy to (3),

$$
\left(l_{2}^{\alpha}\right)^{\bullet}=\partial_{j} v_{i} l_{i}^{\alpha}
$$


When the continuum and its stress field perform a rigid body motion, the material derivative

$$
\left(\sigma_{i j} l_{i}^{\alpha} l_{j}^{\beta}\right)^{\bullet}=\left(\sigma_{i j}+\sigma_{k i} \partial_{i} v_{k}+\sigma_{i k} \partial_{j} v_{k}\right) l_{i}^{\alpha} l_{i}^{\beta}
$$

vanishes. In view of the symmetry of the stress tensor, this remark yields the following definition of stress rate, which is due to Cotter and Rivlin [5]:

$$
\sigma_{i j}^{\prime \prime}=\sigma_{i i}^{\cdot}+\partial_{i} v_{k} \sigma_{k i}+\partial_{j} v_{k} \sigma_{k i} \text {. }
$$

Note that the right-hand side of (14) has the same structure as the expression in the bracket in (5) which can be written as

$$
\left(\partial_{(i} v_{j)}\right)^{\prime \prime}=\left(\partial_{(i} v_{j)}\right)^{\bullet}+\partial_{i} v_{k} \partial_{(k} v_{j)}+\partial_{j} v_{k} \partial_{(k} v_{i)} .
$$

Cotter and Rivlin [5] have shown how higher rates of stress can be obtained by a continuation of the procedure that led to (14). While the definition (14) lends itself more readily to this generalization than the definition (11), it has the drawback that $\sigma_{i j}^{\prime \prime}=0$ does not imply stationary behavior of the stress invariants.

5. Oldroyd's definition. Let the stress tensor at the particle $P$ and the time $t$ be written as a linear combination of the dyadic products of the vectors $l_{i}^{\alpha}$ :

$$
\sigma_{i j}=c_{\alpha \beta} l_{i}^{\alpha} l_{i}^{\beta} .
$$

On account of (12), the coefficients $c_{\alpha \beta}$ of this combination will be stationary if

$$
\begin{aligned}
\sigma_{i j}^{\circ} & =s_{\alpha \beta} \partial_{k} v_{i} l_{k}^{\alpha} l_{i}^{\beta}+s_{\alpha \beta} \partial_{k} v_{j} l_{i}^{\alpha} l_{k}^{\beta} \\
& =\sigma_{k i} \partial_{k} v_{i}+\sigma_{i k} \partial_{k} v_{j}
\end{aligned}
$$

In view of the symmetry of the stress tensor, it follows from this remark that

$$
\sigma_{i i}^{\prime \prime \prime}=\sigma_{i i}^{\cdot}-\sigma_{i k} \partial_{k} v_{i}-\sigma_{i k} \partial_{k} v_{i}
$$

is an acceptable definition of stress rate.

This definition, which is due to Oldroyd [6], has the same disadvantage as that of Cotter and Rivlin: the stress invariants need not be stationary if $\sigma_{i j}^{\prime \prime \prime}$ vanishes.

6. Truesdell's definition. The infinitesimal force transmitted across the oriented surface element $d A_{i}$ is

$$
d P_{i}=\sigma_{i i} d A_{i} .
$$

Let this force be written as a linear combination of the vectors $l_{i}^{\alpha}$ :

$$
d P_{i}=d C_{\alpha} l_{i}^{\alpha} .
$$

According to (12), the coefficients $d C_{\alpha}$ of this combination will be stationary if

$$
\left(d P_{i}\right)^{\bullet}=d C_{\alpha} \partial_{k} v_{j} l_{k}^{\alpha}=d P_{k} \partial_{k} v_{i} .
$$

Substitution of (20) into (21) and use of (7) furnishes $\sigma_{i j}^{\prime \prime \prime} d A_{i}=0$, where

$$
\sigma_{i j}^{\prime \prime \prime \prime}=\sigma_{i i}^{\bullet}+\sigma_{i j} \partial_{k} v_{k}-\sigma_{i k} \partial_{k} v_{i}-\sigma_{i k} \partial_{k} v_{i} .
$$

As follows from its derivation, Eq. (22) represents an acceptable definition of stress rate.

This definition, which is due to Truesdell [2, p. 604], may seem more far-fetched than the preceding ones, because it is based on the consideration of elementary forces rather than stresses. It can be shown, however, that Truesdell's stress rate is closely 
related to the partial derivative of Kirchhoff's stress tensor with respect to time, the other independent variables being the rectangular Cartesian coordinates of the particles in some reference state (see [7], Chap. IX, Sec. 4). Note that the vanishing of Truesdell's stress rate does not imply stationary behavior of the stress invariants.

7. Use in constitutive equations of plasticity. In the theory of perfectly plastic solids, a state of stress is said to be at or below the yield limit according to whether a certain function of the stress invariants, the yield function, is zero or negative. States of stress that furnish positive values of the yield function cannot be supported by these solids.

The constitutive equations describing elastic, perfectly plastic behavior are generally conceived as resulting from the superposition of the rates of deformation of an elastic and a rigid, perfectly plastic constituent. To facilitate this superposition, the constitutive equation of the first constituent is usually assumed to establish a one-to-one correspondence between the rate of deformation and the stress rate. The second constituent is supposed to be rigid under stresses below the yield limit, and to flow plastically under stresses at the yield limit.

In the composite elastic, perfectly plastic solid two criteria are therefore used to judge a given variation of stress in time: the stress rate in the elastic constituent, and the rate of change of the yield function in the plastic constituent. To avoid contradictions, the yield function should be stationary when the stress rate vanishes. A similar condition arises in the theory of elastic, work-hardening solids, where a vanishing stress rate should go with a stationary state of hardening. Only Jaumann's definition of stress rate satisfies these conditions. For use in the constitutive equations of plasticity, Jaumann's definition is therefore preferable to the other definitions of stress rate discussed in this paper.

\section{Biblography}

1. R. S. Rivlin and J. L. Ericksen, J. Ratl. Mech. Anal. 4, 323 (1955)

2. C. Truesdell, J. Ratl. Mech. Anal. 2, 593 (1953)

3. G. Jaumann, Grundlagen der Bewegungslehre, Leipzig, 1905; see also Sitz. ber. Akad. Wiss. Wien (IIa) 120, 385 (1911)

4. H. Fromm, Ingenieurarchiv 4, 452 (1933); S. Zaremba, Mémorial Sci. Math. No. 82, Paris, 1937; T. Y. Thomas, Proc. Nat. Ac. Sci. 41, 762 (1955); W. Noll, J. Ratl. Mech. Analysis 4, 3 (1955); R. Hill, J. Mech. Phys. Solids 7, 209 (1959)

5. B. A. Cotter and R. S. Rivlin, Quart. Appl. Math. 13, 177 (1955)

6. J. G. Oldroyd, Proc. Roy. Soc. (A)200, 523 (1950); See also A. E. Green, Proc. Roy. Soc. (A)234, 46 (1956)

7. W. Prager, Einführung in die Kontinuumsmechanik, Birkhäuser, Basel, 1960 